\title{
Monitoring the effect of asymmetrical vertical strain on Janus single layers of MoSSe via vibrational spectrum
}

\author{
A. Kandemir, ${ }^{1, a)}$ F. M. Peeters, ${ }^{2}$ and H. Sahin ${ }^{3,4}$ \\ ${ }^{1}$ Department of Materials Science and Engineering, Izmir Institute of Technology, 35430 Izmir, Turkey \\ ${ }^{2}$ Department of Physics, University of Antwerp, Groenenborgerlaan 171, B-2020 Antwerpen, Belgium \\ ${ }^{3}$ Department of Photonics, Izmir Institute of Technology, 35430 Izmir, Turkey \\ ${ }^{4}$ ICTP-ECAR Eurasian Center for Advanced Research, Izmir Institute of Technology, 35430 Izmir, Turkey
}

(Received 6 June 2018; accepted 9 August 2018; published online 30 August 2018)

Using first principles calculations, we study the structural and phononic properties of the recently synthesized Janus type single layers of molybdenum dichalcogenides. The Janus MoSSe single layer possesses $2 \mathrm{H}$ crystal structure with two different chalcogenide sides that lead to out-of-plane anisotropy. By virtue of the asymmetric structure of the ultra-thin Janus type crystal, we induced the out-of-plane anisotropy to show the distinctive vertical pressure effect on the vibrational properties of the Janus material. It is proposed that for the corresponding Raman active optical mode of the Janus structure, the phase modulation and the magnitude ratio of the strained atom and its first neighbor atom adjust the distinctive change in the eigen-frequencies and Raman activity. Moreover, a strong variation in the Raman activity of the Janus structure is obtained under bivertical and univertical strains. Not only eigen-frequency shifts but also Raman activities of the optical modes of the Janus structure exhibit distinguishable features. This study reveals that the vertical anisotropic feature of the Janus structure under Raman measurement allows us to distinguish which side of the Janus crystal interacts with the externals (substrate, functional adlayers, or dopants). Published by AIP Publishing. https://doi.org/10.1063/1.5043207

The isolation of graphene as a one-atomic layer of graphite has led to an enormous increase in the research field of ultra-thin materials. ${ }^{1,2}$ Two-dimensional (2D) transition metal dichalcogenides (TMDs) are an important family of ultra-thin materials owing to extraordinary electronical and optical properties such as the gap in the visible frequency range and the good carrier mobility at room temperature. ${ }^{3-10}$

The interaction between external effects (such as substrate, functional adlayers, or dopants) and ultra-thin TMDs is substantially different from that of their bulk version. ${ }^{11,12}$ The properties of ultra-thin TMDs can be tailored by growing them on different substrates. ${ }^{13}$ Lee et al. have shown that monolayers of layered TMDs are shaped diversely by interacting with different substrates like silicon, sapphire, quartz, graphene, h-BN, and $\mathrm{BiFeO}_{3}{ }^{14}$ Besides, the flower-shaped $\mathrm{MoS}_{2}$ on graphene oxide thin films ${ }^{15}$ and the dendrite morphology of $\mathrm{MoS}_{2}$ on silicon carbide ${ }^{16}$ and on $\mathrm{LaAlO}_{3}{ }^{17}$ have been achieved owing to particular interaction between the substrate and the TMD. Tellurium compounds of the TMDs can be reconstructed into two different crystal phases, distorted $1 \mathrm{~T}$ and $2 \mathrm{H}$ phases, due to external effects like strain induced by the substrate or excessive energy (heat or electric field). ${ }^{18-23}$ In addition, the vibrational properties of ultra-thin materials allow us to characterize the material and to determine its phase; however, the substrate and external dopants can distort the measurement. Effects of the substrate on the Raman active

a)Electronic mail: alikandemir@iyte.edu.tr modes of single layers of $\mathrm{MoS}_{2}$ have been extensively investigated by Buscema et al., and it was shown that it leads to shifts in the frequency and may also result in a change of the Raman intensity of the Raman active modes. ${ }^{24}$ Besides, Mlack et al. observed the same phenomena for monolayer $\mathrm{WS}_{2}$ and found frequency shifts before and after the transfer process. ${ }^{25}$

Furthermore, recently synthesized Janus type TMD, i.e., single layer of MoSSe ${ }^{26,27}$ exhibits remarkable asymmetry in the crystal structure. The two different chalcogenide sides of Janus type 2D structures are expected to behave differently when put on surfaces. It is an open question how external effects from substrate amend properties of Janus materials. In this work, the induced out-of-plane anisotropy effects on the vibrational and structural properties of single layers of Janus MoSSe are investigated by applying vertical strain on chalcogenide atoms.

To determine the optimized crystal structure and ground state electron density, first-principle calculations were performed using the Vienna ab initio Simulation Package (VASP) ${ }^{28,29}$ implemented with the-plane-wave basis projector-augmented wave (PAW) method. The electronic exchange-correlation part is treated within the generalized gradient approximation (GGA) in the Perdew-Burke-Ernzerhof (PBE) form applied. ${ }^{30}$ For van der Waals (vdW) correction, the density functional theory (DFT)-D2 method of Grimme was inserted to the GGA functional. ${ }^{31}$

In all the calculations, a plane-wave basis set with kinetic energy cutoff of $800 \mathrm{eV}$ was used. For the convergence 
criterion, the total energy difference between the sequential steps in the calculations was taken to be $10^{-8} \mathrm{eV}$. The total force in the unit cell was reduced to a value of less than $10^{-7} \mathrm{eV} / \AA$. To hinder interactions between the adjacent cells, at least $14 \AA$ vacuum spacing was used along the $z$-direction. Because of the importance of spin orbit interactions in the TMD structure, all calculations were spin polarized. The charge transfer analysis in the structure was determined by the Bader technique. ${ }^{32}$ The vibrational properties were obtained via the PHONOPY code $^{33}$ that uses as input the force constants calculated by the finite-displacement method.

The structure of the Janus MoSSe single layer that possesses the $2 \mathrm{H}$ crystal phase is shown in Fig. 1(a). As seen in the figure, the Janus structure consists of separated atomic layers of sulphur, molybdenum, and selenium atoms. The lattice constant of the Janus crystal structure is calculated to be $a=b$ $=3.25 \AA$. The corresponding Mo-S and Mo-Se bond lengths are $d_{\mathrm{Mo}-\mathrm{S}}=2.42 \AA$ and $d_{\mathrm{Mo}-\mathrm{Se}}=2.53 \AA$, respectively. The vertical distance between the chalcogenide $S$ and $\mathrm{Se}$ atoms taken as the thickness of the Janus monolayer is found to be $3.23 \AA$. Bader charge analysis shows that the charge transfer from transition metal atoms to the chalcogenide atoms is different: 0.5 and $0.4 e^{-}$charges are donated to $\mathrm{S}$ and $\mathrm{Se}$ atoms by the Mo atom.

The dynamical stability of the Janus MoSSe single layer is shown in the phonon band diagram [Fig. 1(b)]. The unit cell of the Janus structure contains three atoms [Fig. 1(a)]; therefore, the phonon dispersion consists of 3 acoustic and 6 optical modes where the vibration representation of the optical modes is described as $\Gamma=2 \mathrm{E}^{\prime}+2 \mathrm{E}^{\prime \prime}+2 \mathrm{~A}_{1} \cdot{ }^{34} \mathrm{As}$ seen in Fig. 1(b), the frequencies of the doubly degenerate in-plane optical modes, $\mathrm{E}^{\prime \prime}$ and $\mathrm{E}^{\prime}$, are 200 and $344 \mathrm{~cm}^{-1}$, respectively. Besides, two $\left(\mathrm{A}_{1}\right)$ singly degenerate out-of-plane optical modes are split as low-frequency $\mathrm{A}_{1}^{L}$ and high frequency $\mathrm{A}_{1}^{H}$ modes, which are 286 and $432 \mathrm{~cm}^{-1}$, respectively. The calculated phonon frequencies of the Janus single layer are in agreement with the experimental and theoretical results. 26,35

Unlike the well-known TMDs such as $\mathrm{MoS}_{2}, \mathrm{WS}_{2}$, and $\mathrm{WSe}_{2}$, the response of Janus TMDs against vertical strain can be expected to be quite different due to out-of-plane anisotropy. The vertical component of strain dominates for ultra-thin materials, and therefore, vertical strain effects on the vibrational properties of the Janus MoSSe single layer can be exploited to reveal the asymmetric feature of the Janus structure.

To begin with, the case of both chalcogenide sides of the Janus MoSSe single layer that experiences compressive and tensile strain is considered. Figure 2 shows the effect of bivertical strain on the vibrational and structural properties of the Janus crystal. First of all, our Raman activity calculations show that all optical modes are Raman active and three prominent Raman peaks are obtained in the $\mathrm{A}_{1}^{L}, \mathrm{E}^{\prime}$, and $\mathrm{A}_{1}^{H}$ optical modes. Besides, corresponding eigenvectors of the optical modes of the Janus structure and the normalized eigenvector magnitude of each atom are shown in Fig. 2. Subsequently, 1 and 2 percent bivertical compressive and tensile strain are applied to the Janus structure. As expected in the optical modes of the Janus structure, a strong phonon hardening is observed under compressive strain due to the suppression in the Mo-chalcogenide bond distances, but on the other hand, clear phonon softening is seen under tensile strain due to the increase in the Mo-chalcogenide bond distances. It is also found that vertical strain is purely ineffective to change the planar component of the Mo-chalcogenide bond distances. Further analysis on the change in eigen-frequencies shows that low-frequency optical modes $\left(\mathrm{A}_{1}^{L}\right.$ and $\left.\mathrm{E}^{\prime \prime}\right)$ have $\sim 1.8 \mathrm{~cm}^{-1} /$ strain rate of change, whereas high-frequency optical modes $\left(\mathrm{A}_{1}^{H}\right.$ and $\left.\mathrm{E}^{\prime}\right)$ have $\sim 2.3 \mathrm{~cm}^{-1} /$ strain rate of change.

In addition to the Raman shifts of the strained Janus structures, the Raman activity trends of the optical modes under bivertically strain are shown in Fig. 2(a). Since there are no external effects (at the ground state), $E^{\prime}$ and $A_{1}^{L}$ have the two-highest Raman activities that can be seen in Raman measurement. As seen in Fig. 2(a), there is a smooth decreasing trend at the Raman activity of $\mathrm{A}_{1}^{L}$ optical mode under bivertical squeezing. On the other hand, Raman activities of the other optical modes have increasing trend under bivertical compression. It is worthwhile to note that $\mathrm{A}_{1}^{L}$ is the most prominent Raman peak under tensile strain and $\mathrm{E}^{\prime}$ is the most prominent Raman peak under compressive strain. Moreover, $\mathrm{A}_{1}^{H}$ becomes one of the two-highest Raman active modes under bivertical squeezing. Besides, it is also found that the highest optical mode as frequency, $\mathrm{A}_{1}^{H}$, disappears when the applied tensile strain increases.
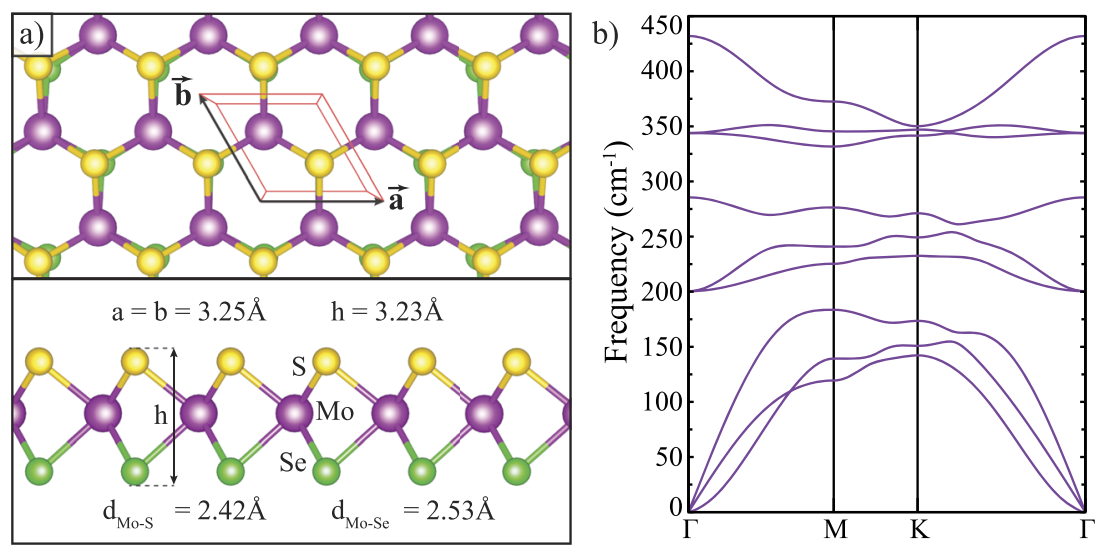

FIG. 1. (a) Top (perspective) and side views of the geometric structure of the Janus MoSSe single layer. Violet, yellow, and green balls show Mo, S, and Se atoms, respectively. (b) Phonon band diagram of the Janus MoSSe single layer. 

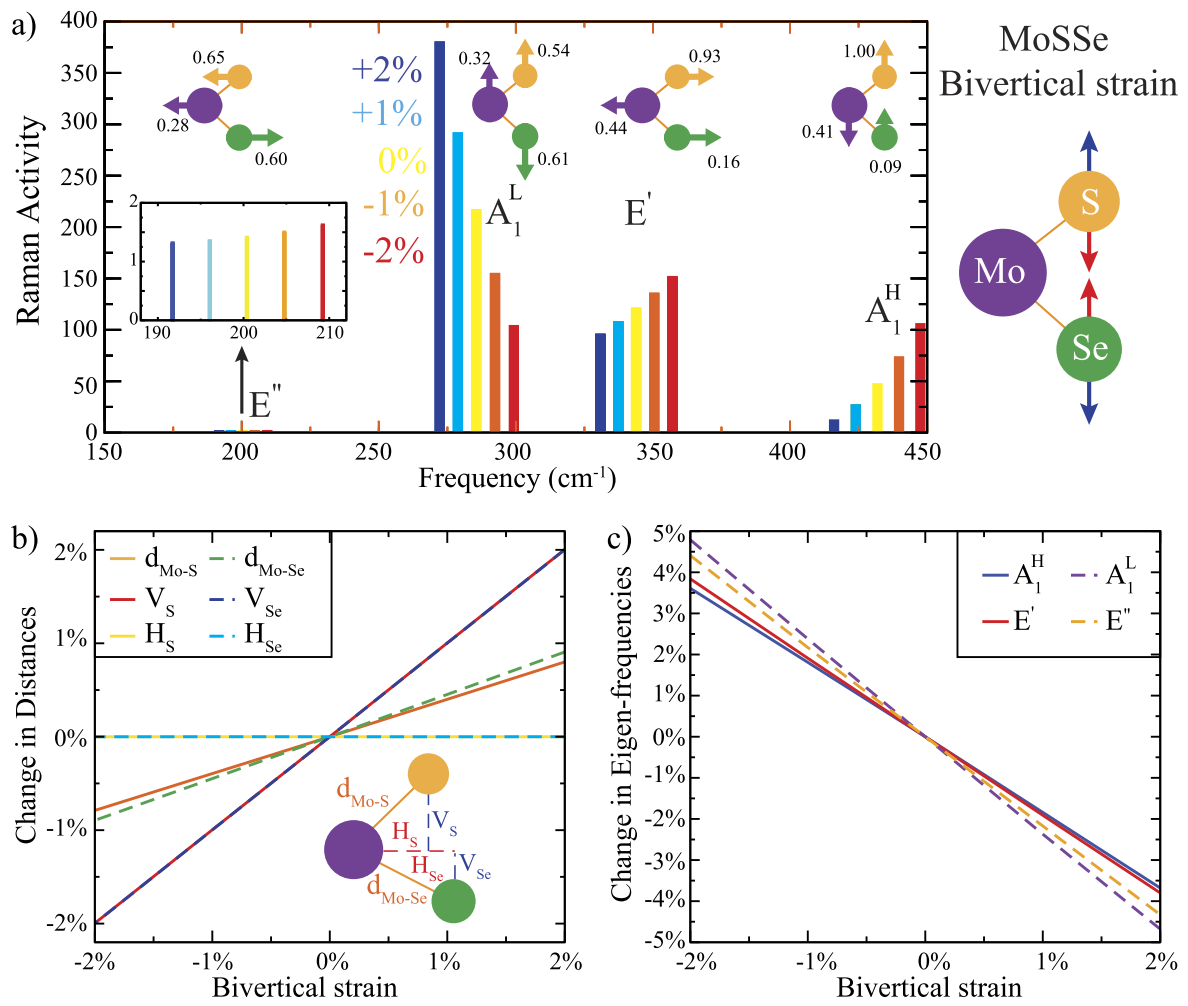

FIG. 2. The effect of bivertical strain on the vibrational and structural properties of the Janus MoSSe structure. (a) Raman activity as a function of frequency and strain. (b) Change in Mo-chalcogenide bond distances with horizontal and vertical components. (c) Shift in eigen-frequencies of the Janus structure as percentage. Corresponding eigenvectors of the optical modes with the magnitudes are shown in the inset. The magnitudes of eigenvector of each atom are normalized according to the highest one.

Next, vertical strain on the $\mathrm{S}$ side of the Janus MoSSe single layer is investigated. The vibrational and structural properties under vertical strain on the $S$ side of the single layer Janus structure are shown in Fig. 3. As seen, the asymmetric structure effect exists, and specific behavior in the vibrational properties of the Janus structure is observed. The low-frequency optical modes remain unchanged under vertical strain; however, the high-frequency optical modes exhibit phonon hardening and softening under compressive and tensile vertical strain, respectively. The corresponding eigenvectors of the low-frequency optical modes of the Janus structure point that the strained S atom performs in-phase motion with the Mo atom, whereas
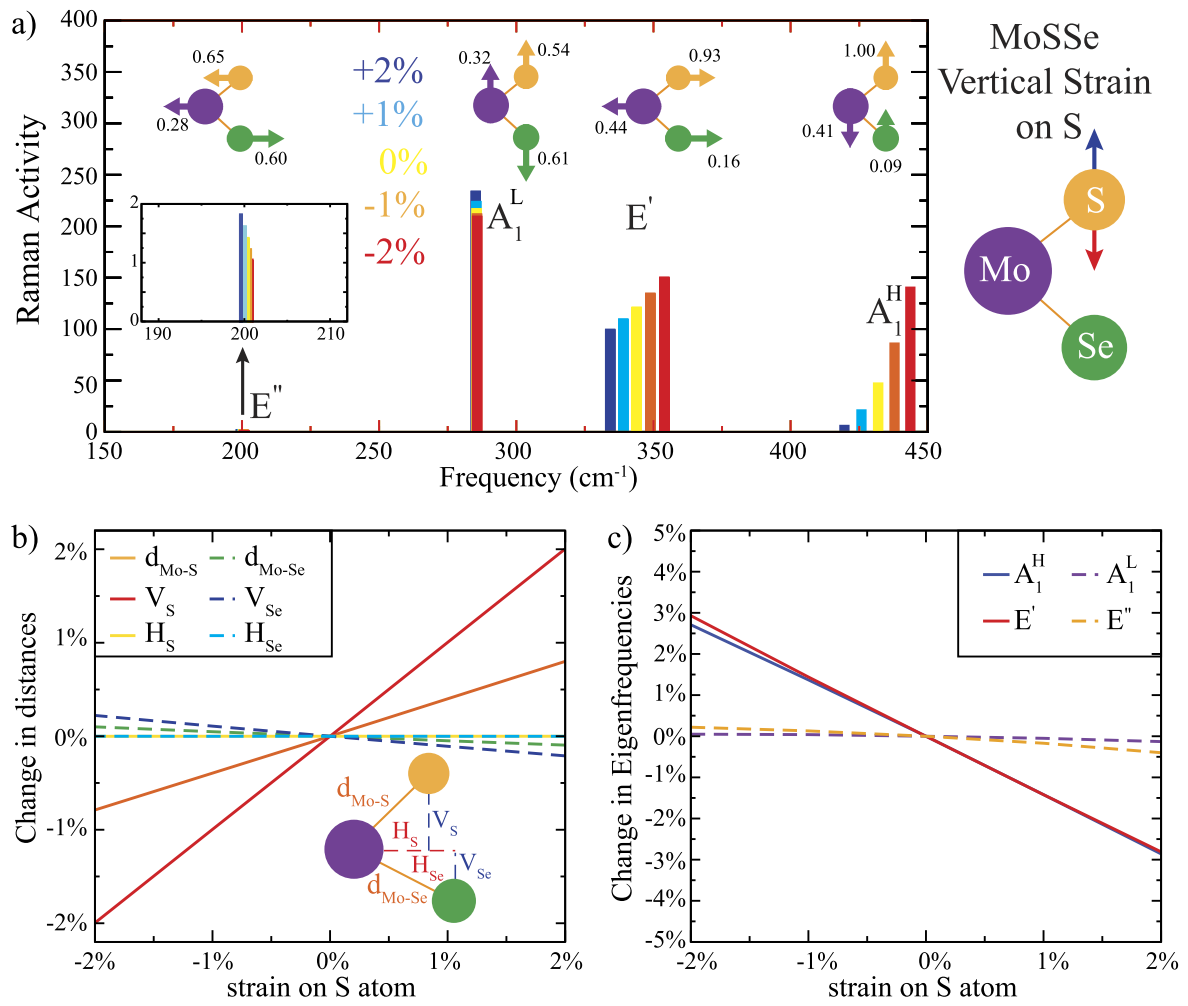

FIG. 3. The effect of vertical strain along the $\mathrm{S}$ side on the vibrational and structural properties of the Janus MoSSe structure. (a) Raman activity as a function of frequency and strain. (b) Change in Mo-chalcogenide bond distances with horizontal and vertical components. (c) Shift in eigen-frequencies of the Janus structure as percentage. Corresponding eigenvectors of the optical modes with the normalized magnitudes are shown in the inset. The magnitudes of eigenvector of each atom are normalized according to the highest one. 

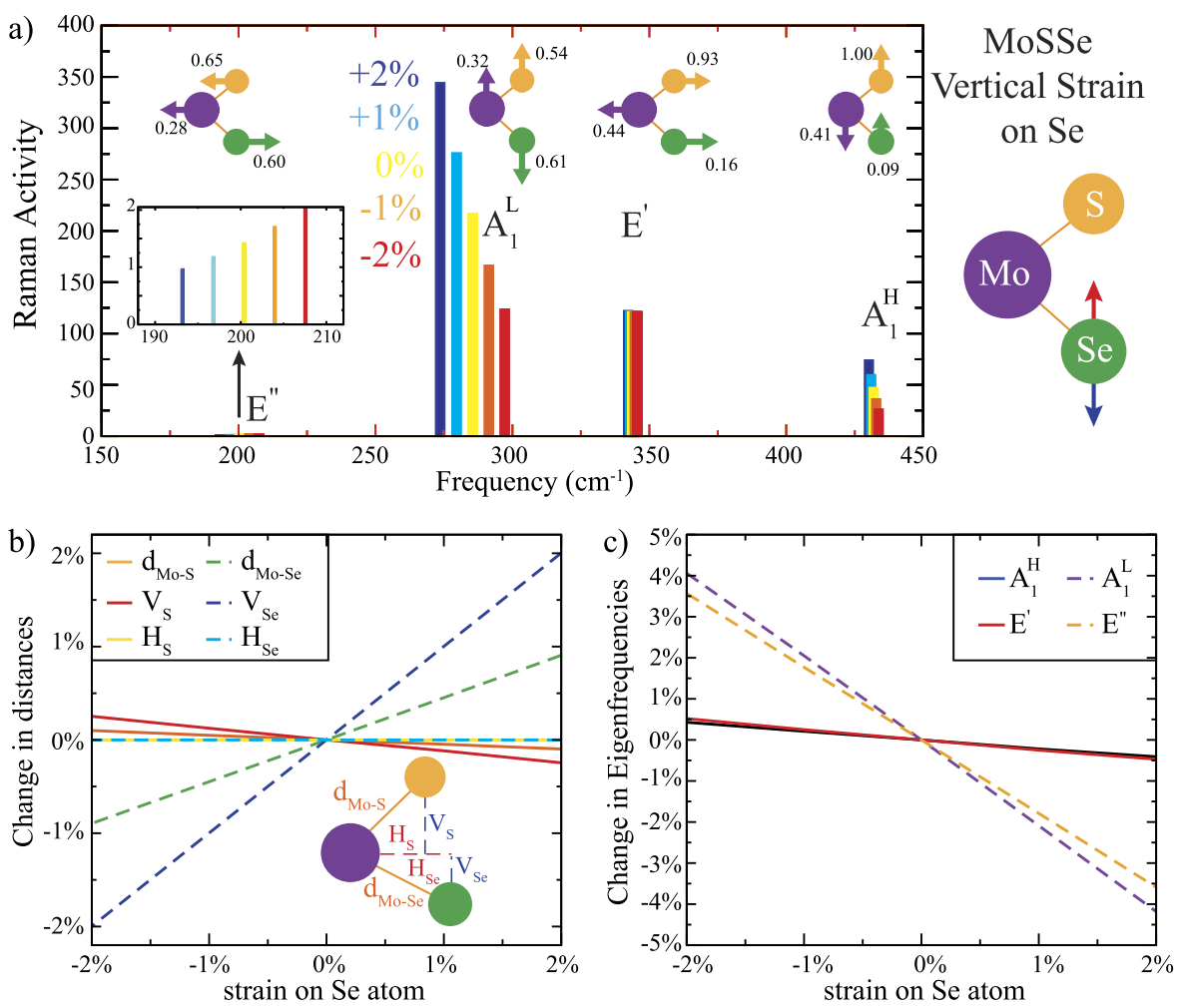

FIG. 4. The effect of vertical strain along the Se side on the vibrational and structural properties of the Janus MoSSe structure. (a) Raman activity as a function of frequency and strain. (b) Change in Mo-chalcogenide bond distances with horizontal and vertical components. (c) Shift in eigen-frequencies of the Janus structure as percentage. Corresponding eigenvectors of the optical modes with the normalized magnitudes are shown in the inset. The magnitudes of eigenvector of each atom are normalized according to the highest one. those of the high-frequency optical modes show out-of-phase motion. Therefore, the phase contrast in the corresponding eigenvectors between strained and its first neighbor atom determines the shift in the eigen-frequency. The fact remains that not only the phase contrast but also the increased magnitude of eigenvector of the strained chalcogenide with respect to its first neighbor atom articulates the shift in the eigen-frequency. The rate of change of the high-frequency optical modes is found to be $\sim 1.5 \mathrm{~cm}^{-1} /$ strain.

Furthermore, different trends are seen in the Raman activity of the optical modes under vertical strain on the $\mathrm{S}$ side. As seen in Fig. 3(a), change in the Raman activities of the lowfrequency optical modes is insignificant and shows no measurable trend compared to the high-frequency optical modes as seen in the eigen-frequency shifts. On the other side, significant change is seen in the Raman activities of the high-frequency optical modes, which decreases since the vertical tensile strain on the $\mathrm{S}$ side increases. Even though the $\mathrm{A}_{1}^{L}$ optical mode is inactive under the vertical strain, it is the most prominent Raman peak in all conditions. Moreover, the strongly measurable Raman activity trend is seen at the high-frequency optical modes. In all circumstances, the $\mathrm{E}^{\prime}$ to $\mathrm{A}_{1}^{H}$ Raman activity ratio has the descent behavior under compressively vertical strain on S. Besides, for bivertical strain, the same trend is seen at the highest $\mathrm{A}_{1}^{H}$ optical mode.

In addition, vertical strain on the Se side of the Janus MoSSe single layer is considered. The vibrational and structural properties under vertical strain on the Se side of the single layer Janus structure are shown in Fig. 4. A very distinct behavior is found as compared to the strained $\mathrm{S}$ side case. Now, the low-frequency optical modes exhibit phonon hardening and softening under vertical strain on the Se side, and this is understandable as long as there is out-of-phase motion and high eigenvector magnitude difference between strained chalcogenide and its first neighbor atom. However, negligible change in the high-frequency optical modes is obtained under vertical strain on the Se side of the single layer Janus structure. As expected, the much lower eigenvector magnitude of the strained atom than that of its first neighbor atom is the reason of the slight change in the eigen-frequency of the high frequency optical modes. Notice that a higher $(\sim 1.9$ $\mathrm{cm}^{-1} /$ strain) rate of change is found for eigen-frequency shift in the low-frequency optical modes.

Moreover, Fig. 4(a) shows the Raman activity trends of the optical modes under vertical strain on the Se side. First, it is seen that the Raman activity of the $\mathrm{E}^{\prime}$ optical mode is insensitive to the vertical strain on the Se side. Second, the other high-frequency optical mode, $\mathrm{A}_{1}^{H}$, shows decreasing trend under bivertical compressive strain. As in the vertical strain on the $\mathrm{S}$ case, the $\mathrm{E}^{\prime}$ to $\mathrm{A}_{1}^{H}$ Raman activity ratio is a determiner for the detect vertical strain effect on the Janus structure; however, the rate of change of the $\mathrm{E}^{\prime}$ to $\mathrm{A}_{1}^{H}$ ratio is reversed in the vertical strain on the Se case. Next, Raman activities of the low-frequency optical modes, $\mathrm{E}^{\prime \prime}$ and $\mathrm{A}_{1}^{L}$, show increasing and decreasing trends under bivertical compressive strain, respectively. Furthermore, it is seen that at high tensile strain values, $\mathrm{A}_{1}^{L}$ is the highest Raman active mode, whereas $E^{\prime}$ becomes the highest one at high compressive strain values for the Janus structure vertically strained on the Se atom.

In conclusion, the effect of strain on the dynamically stable single layer of the Janus MoSSe crystal was investigated. It was seen that the bivertically strained Janus structure shows phonon hardening and softening, respectively, under compressive and tensile strain due to decrease and increase in the Mochalcogenide bond length distances. A change in the Raman 
activity of the Janus structure was observed under compressive and tensile strain. When we induce out-of-plane anisotropy, the obtained changes in the vibrational spectrum are understood by noticing that the high-frequency optical modes of the Janus single layer are in the pocket of the sulphur side, whereas the selenide side is responsible for the low-frequency optical modes. It was found that the phase of the vibrations of the atoms in the Janus structure is a primary factor for the distinctive feature in the optical modes; furthermore, the ratio of the eigenvector magnitude between the pressure induced side and first neighbor side is affected. Besides, not only eigenfrequency shifts but also Raman activities of the optical modes of the Janus structure show significant trends under both strain along bivertical and univertical cases. Especially, the Raman activity ratio of the high-frequency optical modes is significant identifier for the vertically strained Janus structures. Moreover, Raman activity analysis of the Janus structure showed a strong variation and thus an important experimental probe to distinguish the type of applied strain.

Computational resources were provided by TUBITAK ULAKBIM, High Performance and Grid Computing Center (TR-Grid e-Infrastructure). H.S. acknowledges financial support from TUBITAK under Project No. 117F095. F.M.P. was supported by the FLAG-ERA-TRANS $2 D$ TMD.

${ }^{1}$ K. S. Novoselov, A. K. Geim, S. V. Morozov, D. Jiang, Y. Zhang, S. V. Dubonos, I. V. Grigorieva, and A. A. Firsov, Science 306, 666 (2004).

${ }^{2}$ A. K. Geim and K. S. Novoselov, Nat. Mater. 6, 183 (2007).

${ }^{3}$ S. Manzeli, D. Ovchinnikov, D. Pasquier, O. V. Yazyev, and A. Kis, Nat. Rev. Mater. 2, 17033 (2017).

${ }^{4}$ R. A. Gordon, D. Yang, E. D. Crozier, D. T. Jiang, and R. F. Frindt, Phys. Rev. B 65, 125407 (2002).

${ }^{5}$ K. F. Mak, C. Lee, J. Hone, J. Shan, and T. F. Heinz, Phys. Rev. Lett. 105, 136805 (2010).

${ }^{6}$ J. N. Coleman, M. Lotya, A. O’Neill, S. D. Bergin, P. J. King, U. Khan, K. Young, A. Gaucher, S. De, R. J. Smith, I. V. Shvets, S. K. Arora, G. Stanton, H.-Y. Kim, K. Lee, G. T. Kim, G. S. Duesberg, T. Hallam, J. J. Boland, J. J. Wang, J. F. Donegan, J. C. Grunlan, G. Moriarty, A. Shmeliov, R. J. Nicholls, J. M. Perking, E. M. Grieveson, K. Theuwissen, D. W. McComb, P. D. Nellist, and V. Nicolosi, Science 331, 568 (2011).

${ }^{7}$ Q. H. Wang, K. Kalantar-Zadeh, A. Kis, J. N. Coleman, and M. S. Strano, Nat. Nanotechnol. 7, 699 (2012).

${ }^{8}$ H. Sahin, S. Tongay, S. Horzum, W. Fan, J. Zhou, J. Li, J. Wu, and F. M. Peeters, Phys. Rev. B 87, 165409 (2013).

${ }^{9}$ J. S. Ross, P. Klement, A. M. Jones, N. J. Ghimire, J. Yan, D. G. Mandrus, T. Taniguchi, K. Watanabe, K. Kitamura, W. Yao, D. H. Cobden, and X. Xu, Nat. Nanotechnol. 9, 268 (2014).
${ }^{10}$ B. Chen, H. Sahin, A. Suslu, L. Ding, M. I. Bertoni, F. M. Peeters, and S. Tongay, ACS Nano 9, 5326 (2015).

${ }^{11}$ M. Chhowalla, H. S. Shin, G. Eda, L.-J. Li, K. P. Loh, and H. Zhang, Nat. Chem. 5, 263 (2013).

${ }^{12}$ H. Schmidt, F. Giustiniano, and G. Eda, Chem. Soc. Rev. 44, 7715 (2015).

${ }^{13}$ Q. Ji, Y. Zhang, Y. Zhang, and Z. Liu, Chem. Soc. Rev. 44, 2587 (2015).

${ }^{14}$ Y.-H. Lee, L. Yu, H. Wang, W. Fang, X. Ling, Y. Shi, C.-T. Lin, J.-K. Huang, M.-T. Chang, C.-S. Chang, M. Dresselhaus, T. Palacios, L.-J. Li, and J. Kong, Nano Lett. 13, 1852 (2013).

${ }^{15}$ Y. Shi, H. Li, J. I. Wong, X. Zhang, Y. Wang, H. Song, and H. Y. Yang, Sci. Rep. 5, 10378 (2015).

${ }^{16}$ D. Wu, T. Min, J. Zhou, C. Li, G. Ma, G. Lu, M. Xia, and Z. Gu, Sci. Rep. 7, 15166 (2017).

${ }^{17}$ C. Li, Y. Zhang, Q. Ji, J. Shi, Z. Chen, X. Zhou, Q. Fang, and Y. Zhang, 2D Mater. 3, 035001 (2016).

${ }^{18}$ L. Zhou, K. Xu, A. Zubair, A. D. Liao, W. Fang, F. Ouyang, Y.-H. Lee, K. Ueno, R. Saito, T. Palacios, J. Kong, and M. S. Dresselhaus, J. Am. Chem. Soc. 137, 11892 (2015).

${ }^{19}$ J. C. Park, S. J. Yun, H. Kim, J.-H. Park, S. H. Chae, S.-J. An, J.-G. Kim, S. M. Kim, K. K. Kim, and Y. H. Lee, ACS Nano 9, 6548 (2015).

${ }^{20}$ C. H. Naylor, W. M. Parkin, J. Ping, Z. Gao, Y. R. Zhou, Y. Kim, F. Streller, R. W. Carpick, A. M. Rappe, M. Drndic, J. M. Kikkawa, and A. T. C. Johnson, Nano Lett. 16, 4297 (2016).

${ }^{21}$ S. Kim, S. Song, J. Park, H. S. Yu, S. Cho, D. Kim, J. Baik, D.-H. Choe, K. J. Chang, Y. H. Lee, S. W. Kim, and H. Yang, Nano Lett. 17, 3363 (2017).

${ }^{22}$ Z.-Y. Jia, Y.-H. Song, X.-B. Li, K. Ran, P. Lu, H.-J. Zheng, X.-Y. Zhu, Z.-Q. Shi, J. Sun, J. Wen, D. Xing, and S.-C. Li, Phys. Rev. B 96, 041108 (2017).

${ }^{23}$ H. H. Huang, X. Fan, D. J. Singh, H. Chen, Q. Jiang, and W. T. Zheng, Phys. Chem. Chem. Phys. 18, 4086 (2016).

${ }^{24}$ M. Buscema, G. A. Steele, H. S. J. van der Zant, and A. Castellanos-Gomez, Nano Res. 7, 561 (2014).

${ }^{25}$ J. T. Mlack, P. Masih Das, G. Danda, Y.-C. Chou, C. H. Naylor, Z. Lin, N. P. Lopez, T. Zhang, M. Terrones, A. T. C. Johnson, and M. Drndic, Sci. Rep. 7, 43037 (2017).

${ }^{26}$ A.-Y. Lu, H. Zhu, J. Xiao, C.-P. Chuu, Y. Han, M.-H. Chiu, C.-C. Cheng, C.W. Yang, K.-H. Wei, Y. Yang, Y. Wang, D. Sokaras, D. Nordlund, P. Yang, D. A. Muller, M.-Y. Chou, X. Zhang, and L.-J. Li, Nat. Nanotechnol. 12, 744 (2017).

${ }^{27}$ J. Zhang, S. Jia, I. Kholmanov, L. Dong, D. Er, W. Chen, H. Guo, Z. Jin, V. B. Shenoy, L. Shi, and J. Lou, ACS Nano 11, 8192 (2017).

${ }^{28}$ G. Kresse and J. Hafner, Phys. Rev. B 47, 558 (1993).

${ }^{29}$ G. Kresse and J. Furthmuller, Phys. Rev. B 54, 11169 (1996).

${ }^{30}$ J. P. Perdew, K. Burke, and M. Ernzerhof, Phys. Rev. Lett. 77, 3865 (1996).

${ }^{31}$ S. Grimme, J. Comput. Chem. 27, 1787 (2006).

${ }^{32}$ G. Henkelman, A. Arnaldsson, and H. Jonsson, Comput. Mater. Sci. 36, 354 (2006).

${ }^{33}$ A. Togo, F. Oba, and I. Tanaka, Phys. Rev. B 78, 134106 (2008).

${ }^{34}$ A. Kandemir and H. Sahin, Phys. Chem. Chem. Phys. 20, 17380 (2018).

${ }^{35}$ S.-D. Guo, Phys. Chem. Chem. Phys. 20, 7236 (2018). 